Palavras chave: Madeiras da Amazônia

Colorimetria

Espectroscopia de cor Sistema CIELAB

Cor da madeira

Histórico:

Recebido 02/12/2011

Aceito $01 / 10 / 2013$

Keywords:

Amazon wood

Colorimetry

Spectroscopy color

CIELAB system

Wood color

Correspondência: samia_vsb@yahoo.com.br
Sâmia Valéria dos Santos Barros', Graciela Inês Bolzon de Muniz², Jorge Luís Monteiro de Matos $^{2}$

\section{CARACTERIZAÇÃO COLORIMÉTRICA DAS MADEIRAS DE TRÊS ESPÉCIES FLORESTAIS DA AMAZÔNIA}

RESUMO: Objetivou-se, com este trabalho, analisar a variabilidade da cor da madeira nas seções anatômicas (radial, tangencial e transversal) das espécies Breu-vermelho, Tauari-vermelho e Pequiarana, por meio de colorimetria quantitativa, utilizando o sistema CIELAB de cores. As espécies são provenientes da área de exploração florestal da empresa Mil Madeiras Preciosas, localizada no município de Itacoatiara/AM. Foram selecionadas 5 amostras da madeira de cada espécie, sendo determinados os parâmetros colorimétricos: L*, a*, b*, C e h*. Foram realizadas 225 medições no total, por meio do espectrofotômetro Konica Minolta CM-5 acoplado ao computador. As espécies Breu-vermelho, Tauari-vermelho e Pequiarana apresentaram diferenças estatísticas nos parâmetros colorimétricos, e baixa saturação na coordenada $\mathrm{a}^{*}$. De acordo com agrupamento de Cluster, a madeira de Breu-vermelho é oliva e/ou rosa-acinzentada, Tauarivermelho é cinza-rosado e Pequiarana é rosa-acinzentado e/ou cinza-rosado. As espécies florestais apresentaram diferença de cor entre os planos anatômicos (radial, tangencial e transversal), sendo influenciados pela coloração amarela, definida no parâmetro b*. A utilização da colorimetria para conhecimento da cor da madeira mostrou-se de aplicação simples e podendo ser empregada desde o desdobro da tora, até aproveitamento final, com possibilidade de agregação de valor ao produto.

\section{COLORIMETRIC CHARACTERIZATION OF THREE WOOD SPECIES FROM THE AMAZON FOREST}

ABSTRACT: The aim of this study was to analyze wood color variability in the (radial, tangential and transversal) anatomic sections of Breu-vermelho, Tauari-vermelho and Pequiarana species through quantitative colorimetry using CIELAB color system. Such species come from a forest sustainable area of Thousand Precious Woods Company, located in Itacoatiara in the Amazon region of Brazil. Five wood samples from each species were selected so as to determine the following colorimetric parameters: $L^{*}$, a*, b*, C e h*. In addition, 225 measurements were carried out with Konica Minolta CM-5 spectrophotometer connected to the computer. Results pointed out to statistical differences in the colorimetric parameters and also a low saturation in $\mathrm{a}^{*}$ in the analyzed species. According to the cluster gathering, Breu-vermelho wood presents olive and/or grayish pink color, Tauari-vermelho is pinkish-gray and Pequiarana is grayish-pink and/ or pinkish-gray. Such species presented differences in color among the three anatomic sections cited above and were also influenced by the yellow color defined in b* parameter. To summarize, colorimetric analysis to establish wood color is a simple procedure which may be used from the sawing of the logs until their final exploitation enabling value aggregation to the final product.
DOI: 10.1590/0104776020142003142 


\section{INTRODUÇÃO}

A cor é uma das características mais importantes para a identificação, classificação e indicação de uso de espécies de madeira, principalmente quando associada aos aspectos de textura e desenho em usos finais com maior valor econômico. Por meio dessa característica, é possível também obter a valorização de espécies pouco conhecidas, fazendo analogias com outras espécies já tradicionais, o que induz a utilização de termos de referência, como "padrão mogno", "padrão cerejeira", entre outros (CAMARGOS; GONÇALEZ, 200I).

A cor também pode ser alterada pelo teor de umidade, pela temperatura, pelas degradações provocadas pelo ataque de organismos xilófagos ou, ainda, pelas reações fotoquímicas dos componentes químicos presentes em sua estrutura (BURTIN et al., 1998; GONÇALEZ et al., 2005; KLUMPERS et al., 1993; ZERBINI, 2008).

De acordo com Möttönen e Kärki (2007) e Nishino et al. (1998), a estrutura anatômica da madeira proporciona enorme variabilidade de cor entre várias espécies, entre diferentes árvores da mesma espécie e, até mesmo, dentro da própria árvore. Camargos e Gonçalez (200I) consideram, que os planos de orientação (transversal, tangencial e radial) influenciam diretamente a cor da madeira.

A colorimetria quantitativa é uma metodologia objetiva e eficaz para a medição da cor da madeira, descrevendo numericamente cada elemento da composição de uma cor por meio de aparelho apropriado (GONÇALEZ et al., 200 I; MORI et al., 2005).

Um dos sistemas mais utilizados para a medição de cores é o sistema CIELAB (Comissão Internacional de lluminantes). Esse sistema é baseado em três elementos: a luminosidade ou claridade, a tonalidade ou matiz e a saturação ou cromaticidade. Esses elementos são obtidos por meio dos parâmetros colorimétricos $L^{*},+a^{*},-a^{*}$, +b*, -b* e C, de acordo com a Figura I.

A luminosidade (variável L*) é definida pela escala cinza, entre o branco e o preto, assume valor 0 para o preto absoluto, e 100 para o branco total. A tonalidade é expressa pelas cores primárias nas coordenadas do eixo horizontal vermelho-verde ( $\left.+\mathrm{a}^{*},-\mathrm{a}^{*}\right)$ e coordenada do eixo vertical amarelo-azul (+b*, -b*) que variam de 0 a 60. A saturação ou cromaticidade (C) é o desvio a partir do ponto correspondente ao cinza no eixo L* (luminosidade). $\mathrm{O}$ ângulo de tinta ( $\left.\mathrm{h}^{*}\right)$ é $\mathrm{o}$ ângulo do círculo derivado dos valores de $\mathrm{a}^{*}$ e $\mathrm{b}^{*}$, variando de 0 a 60 para madeiras (CAMARGOS; GONÇALEZ, 200I; GONÇALEZ et al., 200I).

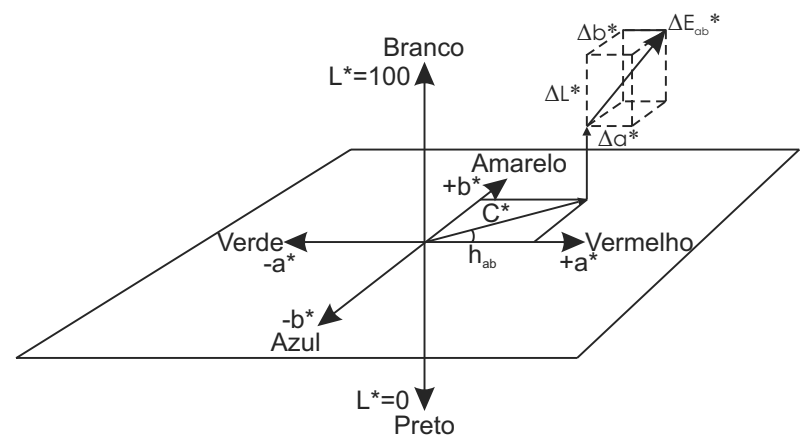

FIGURA I Representação do sistema colorimétrico CIELAB (1976). Fonte: Minolta (1998).

FIGURE I Representation of colorimetric system CIELAB (1976). Source: Minolta (1998).

Nesse contexto, considerando a alta diversidade de espécies florestais na Amazônia, objetivou-se, com este estudo, avaliar a variabilidade da cor da madeira das seções transversal, longitudinal tangencial e longitudinal radial de três espécies florestais da Amazônia, utilizandose a colorimetria quantitativa pelo sistema CIELAB.

\section{MATERIAL E MÉTODOS}

As amostras utilizadas nesse trabalho foram disponibilizadas pelo Laboratório de Tecnologia da Madeira - LTM/UFPR. Os corpos-de-prova são provenientes de amostras (peças) onde foram realizados anteriormente ensaios de flexão. As amostras foram acondicionadas em câmara climática em condições ambientais controladas $\left(20 \pm 2^{\circ} \mathrm{C}\right.$ e $65 \pm 5 \%$ UR) para a manutenção da umidade de equilíbrio.

\section{Localização e descrição da área de coleta}

As espécies florestais são provenientes da área de manejo florestal da empresa Mil Madeiras Preciosas Ltda, localizada no município de Itacoatiara, no estado do Amazonas (BRANDÃO, 2010).

O clima do município é o característico da região tropical, quente, chuvoso e úmido com duas estações bem definidas: Inverno (época de chuvas) e verão (época de calor intenso). A temperatura média anual é de $28^{\circ} \mathrm{C}$.

\section{Identificação das espécies do estudo}

As espécies florestais selecionadas foram identificadas botânicamente, por meio do nome científico no laboratório de física da madeira da Universidade Federal do Amazonas. 
As espécies identificadas foram Tauari-vermelho (Cariniana micrantha Ducke), família Lecythidaceae. É uma espécie arbórea, de altura até $50 \mathrm{~m}$, com tronco de I,5 de diâmetro, com ampla distribuição na Amazônia Brasileira. A madeira é o principal produto da espécie, possui densidade básica de $0,58 \mathrm{~g} \cdot \mathrm{cm}^{-3}$, grã direita, textura média e resistência ao corte manual. Em geral, é comercializada em carpintaria e em marcenaria, tendo grande demanda no mercado interno e externo (INSTITUTO NACIONAL DE PESQUISAS DA AMAZÔNIA - INPA, 2007).

Segundo Matos (2007), a madeira de Caryocar glabrum, da família Caryocaraceae, popularmente conhecida como Pequiarana. Possui madeira pesada, com densidade básica variando de 0,75 a $0,90 \mathrm{~g} \cdot \mathrm{cm}^{-3}$, grã regular e textura média. Os principais usos são para a produção de dormentes, marcenaria, estacas, vigas, caibros, ripas, tábuas, tacos para assoalhos, marcos de portas e janelas, postes, esteios e mourões.

Protium puncticulatum J.F. Macbr. é uma espécie que pertence à Família Burseraceae, conhecida como Breu-vermelho. A madeira apresenta densidade que varia $0,50-0,60 \mathrm{~g} \cdot \mathrm{cm}^{-3}$, sendo classificada como madeira moderadamente pesada. Apresenta grã regular e textura média (TOMAZELLO FILHO et al., 1983).

\section{Amostragem e preparação das amostras}

As amostras de madeira utilizadas são provenientes da coleta de uma árvore por espécie. Foram realizadas medições dos elementos dendrométricos, altura comercial e diâmetro a altura do peito (DAP), com auxílio de fita diamétrica, de acordo com Tabela I.

TABELA I Dados dendrométricos das árvores de Breuvermelho, Pequiarana e Tauari-vermelho.

TABLE I Dendrometric data of Breu-vermelho, Pequiarana and Tauari-vermelho trees.

\begin{tabular}{lccc}
\hline Espécie & DAP $(\mathrm{cm})$ & Altura $(\mathrm{m})$ & QF \\
\hline Breu-vermelho & 55 & 17,64 & $\mathrm{I}$ \\
Pequiarana & 57 & 14,12 & $\mathrm{I}$ \\
Tauari-vermelho & 80 & 14,00 & $\mathrm{I}$ \\
\hline
\end{tabular}

Onde: $\mathrm{DAP}=$ Diâmetro a altura do peito; $\mathrm{QF}=$ Qualidade de fuste. Fonte: Brandão (20I0)

A partir de peças com $5 \times 5 \times 115 \mathrm{~cm}$ utilizadas em ensaios de flexão foram retiradas cinco amostras na dimensão $4 \times 5 \times 10 \mathrm{~cm}$ por espécie, para realizar medições de cor no espectrofotômetro.

\section{Medição da cor}

Para determinação da cor da madeira utilizouse o método CIELAB 1976, no espectrofotômetro Konica Minolta CM-5, acoplado a um computador com programa SpectraMagic NX. O iluminante utilizado foi ○ D65, lâmpada de xenônio difusa que simula a radiação solar diurna, e ângulo de observação $10^{\circ}$. Os dados foram coletados no Laboratório de Anatomia da Madeira da Universidade Federal do Paraná.

A medição da cor foi realizada nas seções transversal, tangencial e radial, de forma aleatoria, sendo realizadas 15 medições em cada uma das amostras, totalizando 225 medições. Não foi realizado qualquer tratamento de superficie, tipo alisamento ou lixamento, para evitar a perda da estabilidade da superficie das espécies.

Os parâmetros colorimétricos obtidos foram L* (luminosidade), a* (coordenada do eixo vermelhoverde), b* (coordenada do eixo azul-amarelo). A medição de cor foi feita na região do espectro visível, no intervalo de 400 a 700 nm, para as três seções.

Para o cálculo dos parâmetros C (saturação) e h* (ângulo de tinta) foram utilizadas as equações I e 2 , segundo o sistema CIELAB 1976.

$$
\begin{aligned}
& \mathrm{C}=\left(\mathrm{a}^{* 2}+\mathrm{b}^{* 2}\right)^{1 / 2} \\
& \mathrm{~h}^{*}=\tan ^{-1}\left(\frac{\mathrm{b}^{*}}{\mathrm{a}^{*}}\right)
\end{aligned}
$$

\section{Análise estatística}

Os valores obtidos dos parâmetros colorimétricos foram comparados estatisticamente pelo teste de Tukey a $5 \%$ de probabilidade para comparação das médias. Os testes foram efetuados no programa Statgraphics Centurion XV.

\section{RESULTADOS E DISCUSSÃO}

$\mathrm{Na}$ Tabela 2, apresentam-se os valores médios e desvio padrão dos parâmetros colorimetricos ( $L^{*}, a^{*}, b^{*}$, $\left.\mathrm{C} \mathrm{e} \mathrm{h}^{*}\right)$ das madeiras das três espécies estudadas.

De acordo com a Tabela 2, observa-se que as madeiras de Breu vermelho, Tauari-vermelho e Pequiarana apresentaram baixo valor de saturação, explicado pela diminuição dos valores das coordenadas $a^{*}$ e b*, variando de 7 a 18 .

Verificou-se baixa predominância do pigmento vermelho $\left(a^{*}\right)$ nas madeiras de Breu-vermelho, Tauarivermelho e Pequiarana. Contudo, o pigmento amarelo 
TABELA 2 Valores médios e desvio padrão (DP) dos parâmetros colorimétricos.

TABLE 2 Average values and standard deviation (DP) of colorimetric parameters.

\begin{tabular}{lcccccccccc}
\hline Espécie & L* & DP & $\mathrm{a}^{*}$ & DP & b* & DP & C & DP & h* & DP \\
\hline Breu-vermelho & $55,72 \mathrm{a}$ & $12,6 \mathrm{I}$ & $7,95 \mathrm{a}$ & $\mathrm{I}, 62$ & $15,1 \mathrm{la}$ & 5,37 & $17,3 \mathrm{la}$ & 4,83 & $60,50 \mathrm{a}$ & 8,79 \\
Tauari-vermelho & $63,04 \mathrm{~b}$ & 11,39 & $7,66 \mathrm{a}$ & 1,67 & $18,43 \mathrm{~b}$ & 5,49 & $20,19 \mathrm{~b}$ & 4,85 & $65,54 \mathrm{~b}$ & 9,17 \\
Pequiarana & $64,67 \mathrm{~b}$ & 10,97 & $7,58 \mathrm{a}$ & 1,73 & $18,87 \mathrm{~b}$ & 5,13 & $20,57 \mathrm{~b}$ & 4,45 & $66,52 \mathrm{~b}$ & 8,68 \\
\hline
\end{tabular}

Médias seguidas pela mesma letra, dentro da mesma coluna, não diferem estatisticamente ao nível de $5 \%$ de probabilidade pelo teste de Tukey.

dado pela coordenada $b^{*}$ exerceu maior influência na caracterização da cor das madeiras de Tauari-vermelho e da Pequiarana. A variável h*, que determina o ângulo de tinta da madeira, confirma a influência do pigmento amarelo nas madeiras de Tauari-vermelho e Pequiarana, colocando-as próximas ao eixo b*, seguida pela coordenada $C$, que também registra essa influência no raio do ângulo. Para a madeira de Breu-vermelho, observa-se maior proximidade ao eixo $\mathrm{a}^{*}$, confirmando maior influência da coloração vermelha na formação da cor desta madeira.

As madeiras das três espécies apresentam claridade $\left(\mathrm{L}^{*}\right)$ média, situando-as próximas de madeiras como o Mogno $(55,48)$ e Eucalipto $(63,62)$, em estudo realizado por Zerbini (2008).

Visualiza-se, ainda, que haja uma tendência de clareamento para as espécies Tauari-vermelho e Pequiarana. Esse clareamento pode ser explicado quantitativamente pelo aumento dos valores de claridade ( $\left.\mathrm{L}^{*}\right)$, que variou de 55 a 64, estes quando próximos de 100 tendem a branco absoluto. E, pela diminuição dos valores da coordenada a* (vermelho), aproximando-se da coordenada $-\mathrm{a}^{*}$, responsável pela coloração verde. Além da coordenada $\mathrm{a}^{*}$, a coordenada $\mathrm{b}^{*}$ tem presença marcante, ajudando a compor a cor mais clara da madeira, produzindo o tom "claro-amarelado" para essas espécies.

Observa-se nas médias que, estatisticamente, apenas no parâmetro a* não houve diferença estatística. Os desvios padrões apresentados na Tabela 2 inferem que a coloração das madeiras das espécies estudadas, de forma geral, é mais homogênea.

Seguindo a proposta apresentada por Camargos e Gonçalez (200I), com a tabela de cores obtida no agrupamento de Cluster para a classificação da cor da madeira, considerando os parâmetros colorimétricos do sistema CIELAB 1976, observou-se para madeira de Breu-vermelho dois tipos de agrupamento de cores, o grupo 09 representado pela cor oliva, e o grupo $22 \mathrm{com}$ rosa-acinzentado, para os valores $L^{*}=55,72 ; a^{*}=7,95$; $b^{*}=15,11 ; C=17,31$ e $h^{*}=60,50$. Para essa madeira, o equilíbrio entre as coordenadas $+a^{*} e+b^{*}$ fortalece na formação de sua cor, por estabelecer o tom mais vermelho na madeira.
A madeira de Tauari-vermelho foi classificada no grupo 16 com a cor cinza-rosado, para valores de $L^{*}=$ 63,$04 ; a^{*}=7,66 ; b^{*}=18,43 ; C=20,19$ e h* $=65,54$. Apesar de existir uma participação equilibrada entre as coordenadas $+a^{*}$ e $+b^{*}$, a coordenada a* também tem presença marcante em função do tipo de pigmento (vermelho) que esta representa na formação da cor desta espécie.

A madeira de Pequiarana também apresentou dois tipos de cor dentro do agrupamento de Cluster, o cinza-rosado no grupo 16 , e rosa-acinzentado no grupo $22\left(L^{*}=64,67 ; a^{*}=7,58 ; b^{*}=18,87 ; C=20,57\right.$ e h* $=66,52)$. A cor da madeira dessa espécie é decorrência da presença marcante da pigmentação amarela, com influência da pigmentação verde.

$\mathrm{Na}$ Tabela 3, apresentam-se os valores médios dos parâmetros colorimétricos ( $\left.L^{*}, a^{*}, b^{*}, C, h^{*}\right)$ das espécies estudadas, considerando as seções radial $(R)$, tangencial $(\mathrm{T})$ e transversal $(\mathrm{X})$.

Verifica-se, na Tabela 3, a diferença significativa das seções tangencial, radial e transversal das madeiras de Breu-vermelho, Tauari-vermelho e Pequiarana. Observase a maior reflectância obtida na região do visível na seção radial em relação à tangencial e transversal. As diferenças significativas entre as seções de observação, dados pelos parâmetros colorimétricos, caracterizam que o sentido de desdobro pode interferir na cor da madeira, pois a coloração é estatisticamente diferente para os três planos de observação.

Observou-se que o plano radial apresentou maior saturação $(C)$ e luminosidade ( $\left.L^{*}\right)$. Como o parâmetro saturação é influenciado pelas coordenadas $a^{*}$ e $b^{*}$, à medida que essas duas variáveis aumentam, os valores da saturação também aumentam. Nesse caso, o maior valor observado foi de 22,39 no plano radial para espécie Tauri-vermelho. Segundo Möttönen e Kärki (2005), estudos microscópicos revelaram que produtos químicos descoloridos na madeira estão concentrados nas células de parênquimas dos raios, o que poderia explicar o fato da seção radial apresentar coloração mais clara, devido à maior proximidade entre as células radiais. 
TABELA 3 Valores médios dos parâmetros colorimétricos dentro de cada tratamento (seção).

TABLE 3 Average values of colorimetric parameters within each treatment (section).

\begin{tabular}{lccccccccccc}
\hline Espécie & $\mathrm{T}$ & $\mathrm{L}^{*}$ & $\mathrm{DP}$ & $\mathrm{a}^{*}$ & $\mathrm{DP}$ & $\mathrm{b}^{*}$ & $\mathrm{DP}$ & $\mathrm{C}$ & $\mathrm{DP}$ & $\mathrm{h}^{*}$ & $\mathrm{DP}$ \\
\hline \multirow{3}{*}{ Breu-vermelho } & $\mathrm{R}$ & $59,13 \mathrm{a}$ & 12,83 & $8,19 \mathrm{ab}$ & $\mathrm{I}, 70$ & $16,25 \mathrm{a}$ & 5,37 & $18,46 \mathrm{a}$ & 4,68 & $61,52 \mathrm{a}$ & 9,18 \\
& $\mathrm{~T}$ & $55,89 \mathrm{a}$ & 13,75 & $8,42 \mathrm{a}$ & 1,83 & $15,64 \mathrm{a}$ & 5,42 & $18,04 \mathrm{a}$ & 4,71 & $59,97 \mathrm{a}$ & 9,72 \\
& $\mathrm{X}$ & $52,13 \mathrm{a}$ & 10,55 & $7,22 \mathrm{~b}$ & 1,03 & $13,44 \mathrm{a}$ & 5,12 & $15,4 \mathrm{la}$ & 4,72 & $60,00 \mathrm{a}$ & 7,62 \\
\hline \multirow{3}{*}{ Tauari-vermelho } & $\mathrm{R}$ & $65,50 \mathrm{a}$ & 11,45 & $8,26 \mathrm{a}$ & 1,82 & $20,52 \mathrm{a}$ & 5,58 & $22,39 \mathrm{a}$ & 4,68 & $66,24 \mathrm{a}$ & 9,61 \\
& $\mathrm{~T}$ & $64,33 \mathrm{a}$ & 12,20 & $7,27 \mathrm{a}$ & 1,84 & $17,59 \mathrm{a}$ & 4,90 & $19,28 \mathrm{a}$ & 4,20 & $65,94 \mathrm{a}$ & 9,50 \\
& $\mathrm{X}$ & $59,27 \mathrm{a}$ & 9,87 & $7,45 \mathrm{a}$ & 1,17 & $17,17 \mathrm{a}$ & 5,58 & $18,90 \mathrm{a}$ & 5,04 & $64,43 \mathrm{a}$ & 8,65 \\
\hline \multirow{3}{*}{ Pequiarana } & $\mathrm{R}$ & $69,14 \mathrm{a}$ & 11,41 & $7,27 \mathrm{~b}$ & 1,66 & $19,52 \mathrm{a}$ & 5,48 & $21,10 \mathrm{ab}$ & 4,62 & $67,63 \mathrm{a}$ & 9,48 \\
& $\mathrm{~T}$ & $3,83 \mathrm{ab}$ & 11,02 & $8,66 \mathrm{a}$ & 1,96 & $19,73 \mathrm{a}$ & 4,04 & $21,78 \mathrm{a}$ & 3,11 & $65,27 \mathrm{a}$ & 8,47 \\
& $\mathrm{X}$ & $61,02 \mathrm{~b}$ & 9,15 & $6,78 \mathrm{~b}$ & 0,78 & $17,36 \mathrm{a}$ & 5,58 & $18,8 \mathrm{lb}$ & 5,00 & $66,65 \mathrm{a}$ & 8,24 \\
\hline
\end{tabular}

Médias seguidas pela mesma letra, dentro da mesma coluna, não diferem estatisticamente ao nível de $5 \%$ de probabilidade pelo teste de Tukey.

Para Nishino et al. (1998) algumas espécies têm diferença de cor e aparência entre as seções tangencial e radial, que podem ser atribuídas às características anatômicas.

A luminosidade $\left(L^{*}\right)$ foi menor no plano transversal em relação aos planos radial e tangencial nas três espécies. No trabalho de Nishino et al. (2000), também foi observado comportamento semelhante na seção transversal, onde as células das fibras podem diminuir a luminosidade, conforme características anatômicas de cada plano. Gonçalez e Gonçalez (200I) também relatam que, na seção transversal, a presença dos anéis de crescimento aumentam o nível de cinza, fazendo com que haja uma diminuição da luminosidade $\left(L^{*}\right)$ e das matizes responsáveis pelas coordenadas $\mathrm{a}^{*} \mathrm{e}$ b*, tornando a madeira mais escura.

O ângulo de tinta $\left(h^{*}\right)$ também apresentou menor valor na seção transversal, em relação às outras duas seções, indicando que o plano transversal é mais escuro que os demais.

A madeira de Breu-vermelho apresentou diferença estatística apenas no parâmetro colorimétrico a*. Considerando a faixa 0 a 60 , e que os valores obtidos variaram de 7 a 8 nos planos analisados. Isso corrobora que essa espécie tende ao clareamento quando exposta a intempérie, uma vez que a pigmentação verde é presença marcante, sendo influenciada pela cor amarela na coordenada $b^{*}$.

A espécie Tauari-vermelho não apresentou diferenças estatísticas em suas médias. Entretanto, observou-se que os valores de a* também variaram entre 7,27 e 8,26, principalmente nos planos tangencial e transversal, influenciados pelos valores do ângulo de tinta $\left(h^{*}\right)$ e de saturação $(C)$, evidenciando a diminuição da coloração.

Diferenças significativas também foram observadas para espécie Pequiarana nos parâmetros colorimétricos $L^{*}, a^{*}$ e $C$, nos planos radial, tangencial e transversal. Embora $\circ$ plano tangencial apresente maior média na pigmentação vermelha, isso não refletiu na claridade da madeira. Dessa forma, entende-se que a forte pigmentação amarela presente nessa espécie, é decisiva na formação de sua cor. Assim, pode-se esperar que a madeira de Pequiarana venha apresentar nuances de pigmentos avermelhados mais vivos na direção tangencial de desdobro. Essa informação é confirmada pelo parâmetro $\mathrm{C}$, que varia proporcionalmente aos valores de $\mathrm{a}^{*} \mathrm{e}^{*}$.

Os valores observados nas espécies amazônicas foram semelhantes a alguns valores encontrados por Nishino et al. (1998) quando estudaram 97 madeiras de espécies da Guiana Francesa, utilizando cinco observações por seção, nas superfícies radial e tangencial. As espécies semelhantes foram Couma guyanensis Aubl., Brosimum utile Pitt., Couratari multiflora Eyma., Protium sagotianum March.

Nesse contexto, ressalta-se a importância na escolha da madeira no momento do desdobro. Quando optar-se por lotes de madeiras mais claras, estas devem ser desdobradas no sentido radial, pois os valores da luminosidade $\left(L^{*}\right)$ neste plano são mais elevados. Da mesma forma, na aquisição de lotes de madeiras mais escuras com corte para o lado tangencial.

Observa-se ainda, que tais informações podem subsidiar uma pré-classificação da madeira, para a combinação de cores em tons variados, considerando a preferência do mercado, cabendo ainda à formação de um banco de dados de cores. 


\section{CONCLUSÕES}

A madeira de Breu-vermelho é caracterizada pela coordenada $a^{*}$ (pigmentação vermelha), com influência da cor amarela na coordenada $b *$, e tendência ao clareamento pelo maior valor de claridade ( $\left.\mathrm{L}^{*}\right)$.

O Tauari-vermelho também é caracterizado pela pigmentação vermelha, com influência dos valores do ângulo de tinta ( $h *$ ) e de saturação (C), evidenciando a diminuição da coloração.

A Pequiarana apresentou forte pigmentação amarela, sendo decisiva na formação de sua cor, com nuances de pigmentos avermelhados mais vivos na direção tangencial, sendo confirmada pelo parâmetro $C$.

As espécies Breu-vermelho, Tauari-vermelho e Pequiarana apresentaram diferenças estatísticas nos parâmetros colorimétricos, baixa saturação na coordenada a*, e claridade ( $\left.\mathrm{L}^{*}\right)$ média.

De acordo com agrupamento de Cluster, a madeira de Breu-vermelho é oliva e/ou rosa-acinzentada, Tauari-vermelho é cinza-rosado, e Pequiarana é rosaacinzentado e/ou cinza-rosado.

As espécies florestais apresentaram diferença de cor entre as seções anatômicas (radial, tangencial e transversal), com maior valor observado no plano radial, sendo influenciadas pela coloração amarela, definida no parametro $b^{*}$.

A utilização da colorimetria quantitativa para conhecimento da cor da madeira revelou-se de aplicação simples e prática, podendo ser empregada na préclassificação da madeira, a partir do desdobro da tora, na qualificação de peças, e na formação de banco de dados.

\section{AGRADECIMENTOS}

Os autores expressam seus agradecimentos ao Laboratório de Tecnologia da Madeira - LTM/UFPR, pela concessão das amostras, e ao Laboratório de Anatomia da Madeira, pelo uso do equipamento na obtenção dos espectros de colorimetria.

\section{REFERÊNCIAS}

BRANDÃO,J. B. Estudo das propriedades físicas e mecânicas de três espécies florestais. 2010. 80 p. Dissertação (Mestrado em Ciências Florestais) - Universidade Federal do Amazonas, Manaus, 2010.

BURTIN, P.; JAY-ALLEMAND, C.; CHARPENTIER, J. P.; JANIN, G. Natural wood colouring process in Juglans sp. (J. nigra, J. regia and hybrid J. nigra $23 \times$ J. regia) depends on native phenolic compounds accumulated in the transition zone between sapwood and heartwood. Trees, Berlin, v. I2, n. 5, p. 258-264, Mar. 1998.
CAMARGOS, J. A. A.; GONÇALEZ, J. C. A colorimetria aplicada como instrumento na elaboração de uma tabela de cores de madeira. Brasil Florestal, Rio de Janeiro, ano 20, n. 7I, p. 30-4I, 200I.

GONÇALEZ, J. C.; JANIN, G.; FARIAS, A. L. S.; GODOI, A. C. L.; CHARRIER, F.; CHARRIER, B. Valorisation d'espèces brésiliennes de substitution pour l'industrie du meuble: tanimbuca et louro. Bois et Forêsts des Tropiques, Paris, v. 286, n. 4, p. 55-64, 2005.

GONÇALEZ, J. C.; JANIN, G.; SANTORO, A. C. S.; COSTA, A. F.; VALLE, A. T. Colorimetria quantitativa: uma técnica objetiva de determinar a cor da madeira. Brasil Florestal, Rio de Janeiro, ano 20, n. 72, p. 47-58, 200 I.

INSTITUTO NACIONAL DE PESQUISAS DA AMAZÔNIA. Castanha-de-macaco: Cariniana micranta Ducke. Manaus, 2007. (Informativo Técnico: Rede de Sementes da Amazônia, 15).

KLUMPERS, J.; JANIN, G.; BECKER, M. The influences of age, extractive content and soil water on wood color in oak: the possible genetic determination of wood color. Annales des Sciences Forestières, Versailles, v. 50, n. I, p. 403-409, 1993.

MATOS, E. H. S. F. Cultivo do pequi e extração do óleo. Brasília: CDT/UnB, 2007. Dossiê técnico.

MINOLTA. Precise color communication: color control from perception to instrumentation. Tokyo, 1998. $59 \mathrm{p}$.

MORI, C. L. S. O.; LIMA, J. T.; MORI, F. A.; TRIGILHO, P. F.; GONÇALEZ, J. C. Caracterização da cor da madeira de clones de híbridos de Eucalyptus spp. Cerne, Lavras, v. I I, n. 2, p. |37-|46, abr./jun. 2005.

MÖTTÖNEN, V.; KÄRKI, T. Effect of drying force on birch wood colour change during high temperature drying. Baltic Forestry, Kaunas, v. I3, n. I, p. |26-| 30, 2007.

NISHINO, Y.; JANIN, G.; CHANSON, B.; DÉTIENNE, P.; GRIL, J.; THIBAUT, B. Colorimetry of wood specimens from French Guiana. Journal of Wood Science, London, v. 44 , p. $3-8,1998$

NISHINO, Y.; JANIN, G.; YAMADA, Y.; KITANO, D. Relations between the colorimetric values and densities of sapwood. Journal of Wood Science, Zurich, v. 46, p. 267-272, 2000.

TOMAZELLO FILHO, M.; CHIMELO, J. P.; GARCIA, P. V. Madeiras de espécies florestais do Estado do Maranhão: II., caracterização Anatômica. IPEF, Piracicaba, n. 23, p. 2936, abr. 1983.

ZERBINI, N. J. Madeiras tropicais com potencial comercial da região do rio Xingu (Pará, Brasil): propriedades tecnológicas e cadeia produtiva. 2008. I87 p. Tese (Doutorado em Engenharia Florestal) - Universidade Federal de Brasília, Brasília, 2008. 